\title{
High Speed PAM -8 Optical Interconnects with Digital Equalization based on Neural
} Network

Gaiarin, Simone; Pang, Xiaodan; Ozolins, Oskars; Jones, Rasmus Thomas; Porto da Silva, Edson; Schatz, Richard; Westergren, Urban; Popov, Sergei; Jacobsen, Gunnar; Zibar, Darko

\section{Published in:}

Proceedings of Asia Communications and Photonics Conference

Link to article, DOI:

10.1364/ACPC.2016.AS1C.1

Publication date:

2016

Document Version

Peer reviewed version

Link back to DTU Orbit

Citation (APA):

Gaiarin, S., Pang, X., Ozolins, O., Jones, R. T., Porto da Silva, E., Schatz, R., Westergren, U., Popov, S., Jacobsen, G., \& Zibar, D. (2016). High Speed PAM -8 Optical Interconnects with Digital Equalization based on Neural Network. In Proceedings of Asia Communications and Photonics Conference https://doi.org/10.1364/ACPC.2016.AS1C.1

\section{General rights}

Copyright and moral rights for the publications made accessible in the public portal are retained by the authors and/or other copyright owners and it is a condition of accessing publications that users recognise and abide by the legal requirements associated with these rights.

- Users may download and print one copy of any publication from the public portal for the purpose of private study or research.

- You may not further distribute the material or use it for any profit-making activity or commercial gain

- You may freely distribute the URL identifying the publication in the public portal 


\title{
High Speed PAM-8 Optical Interconnects with Digital Equalization based on Neural Network
}

\author{
Simone Gaiarin ${ }^{1}$, Xiaodan Pang ${ }^{2}$, Oskars Ozolins ${ }^{2}$, Rasmus Thomas Jones ${ }^{1}$, Edson Porto Da Silva ${ }^{1}$, \\ Richard Schatz ${ }^{3}$, Urban Westergren ${ }^{3}$, Sergei Popov ${ }^{3}$, Gunnar Jacobsen², Darko Zibar ${ }^{1}$ \\ ${ }^{1}$ DTU Fotonik, Technical University of Denmark, 2800 Kgs.Lyngby, Denmark \\ ${ }^{2}$ Acreo Swedish ICT AB, Isafjordsgatan 22, 16440 Kista, Sweden \\ ${ }^{3}$ School of ICT, KTH Royal Institute of Technology, Electrum 229, 16440 Kista, Sweden \\ simga@fotonik.dtu.dk
}

\begin{abstract}
We experimentally evaluate a high-speed optical interconnection link with neural network equalization. Enhanced equalization performances are shown comparing to standard linear FFE for an EML-based 32 GBd PAM-8 signal after 4-km SMF transmission.

OCIS codes: (060.2330) Fiber optics communications, (060.4510) Optical communications
\end{abstract}

\section{Introduction}

Increasing demand for high speed optical interconnects have driven the intensity modulation and direct-detection (IM/DD) systems towards using transceivers of higher bandwidth, lower cost and denser integration. Advanced modulations e.g. pulse amplitude modulations (PAM) together with integrated semiconductor lasers and modulators appear as promising candidates to fulfill the upcoming application requirements [1]. In such systems digital equalization plays an important role to compensate the impairments caused by high-level of integration. For instance, linear equalizers such as feed-forward equalizer (FFE) and decision feedback equalizer (DFE) are commonly employed to reduce/cancel inter-symbol interference (ISI) induced by the limited bandwidth of electro-optic components and fiber chromatic dispersion [2]. However, in a typical IM/DD system employing highly integrated components, due to the correlation of the square-law detection with laser/modulator chirp, fiber dispersion and additive noise, the overall channel response becomes nonlinear and results in difficulty for linear equalizers to perform accurate channel estimations. Recently, studies have shown that machine learning algorithms, including neural network (NN) equalizers, can effectively recover signal from nonlinear distortions, including non-Gaussian additive noise, non-white laser phase noise and fiber nonlinearities in both IM/DD and coherent systems [3-5]. For short range IM/DD systems, simulation studies were performed on a 56 GBd PAM-4 signal, showing promising improvements with respect to the commonly used linear equalizers [6].

In this work, we experimentally evaluate the equalization performance of a neural network-based equalizer to a 32 GBd PAM-8 signal modulated with a broadband high linear integrated electro-absorption modulated laser (EML). We demonstrate that the NN equalizer is able to recover the distorted signal after $4 \mathrm{~km}$ single mode fiber (SMF) transmission. In addition, we compare the equalization performances between the NN equalizer and the conventional symbol-spaced linear FFE implemented with different number of taps, so as to set a benchmark for further detailed explorations.

\section{Neural Network-based Equalizer}

Figure 1 shows the structures of both the conventional linear equalizer based on an FIR filter structure and the NN equalizer applied in this work. In practical system implementation, both equalizers can be firstly trained in a training mode and then operate in a static mode. In a training mode, tap weights $\left(\mathrm{w}_{1}, \mathrm{w}_{2} \ldots \mathrm{w}_{\mathrm{N}}\right)$ of the linear equalizers can

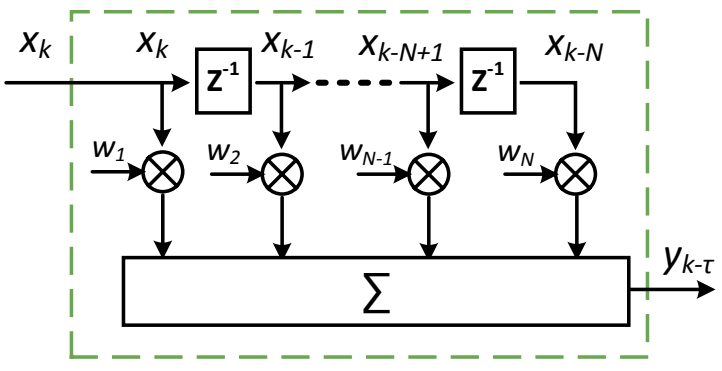

(a)

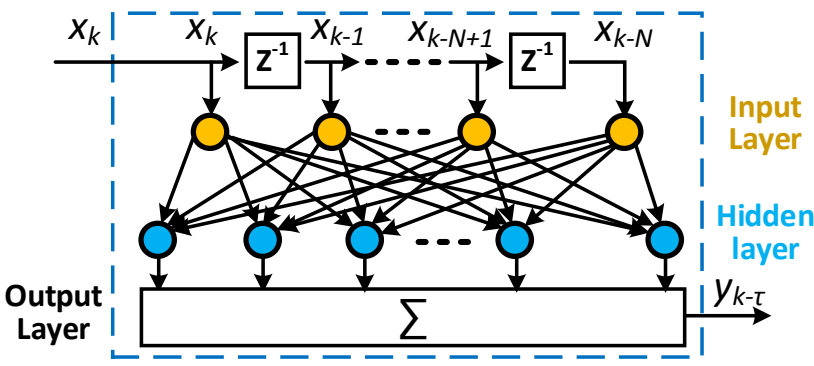

(b)

Fig. 1 Structures of (a) conventional linear equalizer and (b) the neural network equalizer 
be adapted with normalized least-mean-square (NLMS) algorithm to minimize the mean-square error between the received symbols and the training symbols. The NN equalizer is a feed-forward NN with 9 input nodes whose inputs are subsequent samples of the signal. The neural network has a single hidden layer composed by 10 neurons using the hyperbolic tangent as activation function. The network weights were optimized by running the LevenbergMarquardt backpropagation algorithm using a data set of $1.2 \mathrm{M}$ samples, divided in training set, cross validation set and test set with a ratio 70-15-15. For both the equalizers, the output of the network at every instant is $\mathrm{y}_{\mathrm{k}-\mathrm{T}}$ where $\tau$ is a delay between 0 and $\mathrm{N}-1$. Given the fact that the channel is not expected to change over time, after optimizing the tap weights of the linear equalizer, the network weights of the $\mathrm{NN}$ and the delay $T$, the equalizers' structures are kept static for system operation, in such a way that no adaptive equalization is required.

\section{Experimental setup}

The experimental setup is shown in Fig. 2. A pulse pattern generator (PPG) operating at 32GBd and a 3-bit digitalto-analog converter (DAC) with $19 \mathrm{GHz} 3-\mathrm{dB}$ analog bandwidth were used to generate the electrical PAM-8 signal, which consists of multiple delayed patterns of pseudorandom bit sequence with length of $2^{15}-1$ (PRBS 15). The DAC output signal was linearly amplified to $2.2 \mathrm{Vpp}$ prior to modulating a $1550 \mathrm{~nm}$ EML. The EML used in the experiment consists of a high power distribute-feedback (DFB) laser and an InGaAsP travelling wave electroabsorption modulator (TWEAM), both monolithically integrated and with a 3-dB bandwidth of $>100 \mathrm{GHz}$ [7]. The EML is biased at $-1.6 \mathrm{~V}$ with a linear operational swing of the larger than $2 \mathrm{Vpp}$, ensuring low-distortion modulation of the PAM-8 signal. The signal was transmitted over a $4 \mathrm{~km}$ SMF link before reaching the receiver end. The eyediagrams of the optical signal before and after $4 \mathrm{~km} \mathrm{SMF}$ are shown in Fig. 2. Clear eye openings are observed in the optical back-to-back (B2B) case, while the eyes are completely closed after the $4 \mathrm{~km}$ fiber, due to the strong ISI induced by fiber dispersion, which indicates the necessity of digital equalizations.

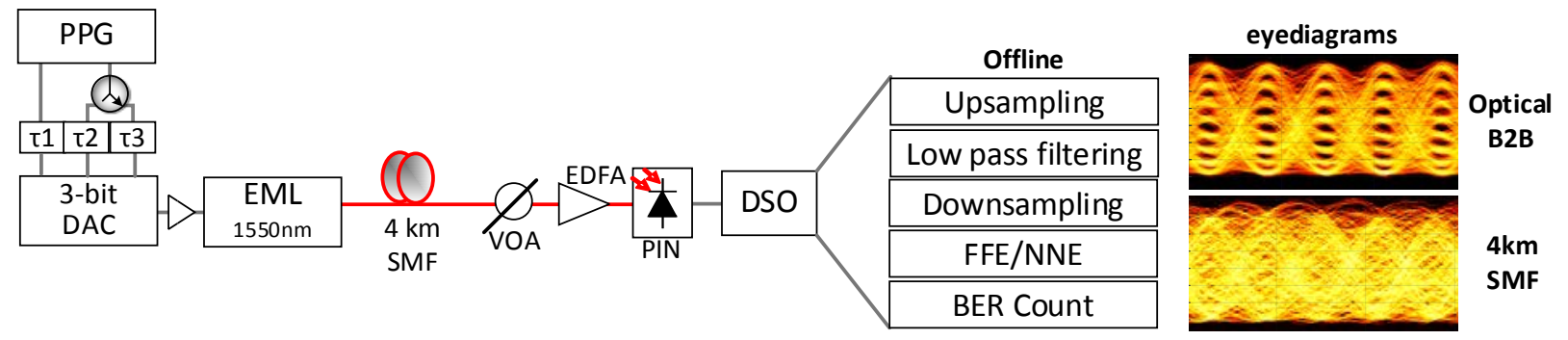

Fig. 2 Experimental setup and signal eyediagrams before/after 4km SMF transmission.

At the receiver, a p-i-n photodiode (PD) with $>90 \mathrm{GHz}$ bandwidth was used to convert the received optical signal to electrical domain. An automatic gain-controlled EDFA with fixed output power was employed as a preamplifier due to the low PD responsivity and the absence of transimpedance amplifier (TIA) in this experiment. We use a variable optical attenuator (VOA) to adjust the input power to the EDFA, which results in an amplified spontaneous emission (ASE) noise dominated scenario. In practical system this receiver structure can be substituted with either a PIN-TIA or avalanche PD (APD) for lower noise and better sensitivity. After PD the signal was sampled at $80 \mathrm{GSa} / \mathrm{s}$ by a $33 \mathrm{GHz}$ real-time digital storage oscilloscope (DSO) and processed offline. The sampled digital signals were firstly resampled to $4 \mathrm{Sa} / \mathrm{Sym}$ to perform low-pass filtering and clock/timing recovery, before being downsampled to $1 \mathrm{Sa} / \mathrm{Sym}$ for equalization. Symbol-spaced FFE with different taps are evaluated as a comparison to the studied NNE. It is noted that other nonlinear functions and number of nodes can be worthy of further investigation. In both cases, the equalizers were firstly trained so the weights and node configurations are adapted to reconstruct the inverse of the (nonlinear) channel response. Then we used the converged configurations as static filters and apply them to all data traces. A total number of $1.2 \mathrm{M}$ bits are used for BER counting.

\section{Results}

Figure 3 presents the BER performances versus received optical power for the 32 GBd PAM-8 in both B2B and after $4 \mathrm{~km}$ SMF transmission cases. A clear error floor above the $3.8 \times 10^{-3}$ BER threshold of $7 \%$-overhead $(\mathrm{OH})$ hard-decision forward error correction (HD-FEC) code is already seen for the B2B case without any equalization. From the eyediagrams of Fig. 2, slight compression can be observed between the 2 lower amplitude levels in the modulated PAM-8 signal. This is due to a trade-off between the linearity and modulation depth for a higher receiver 


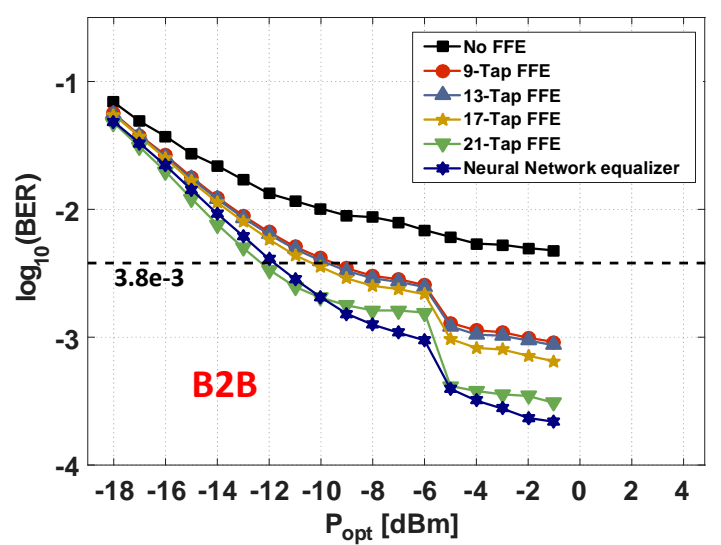

(a)

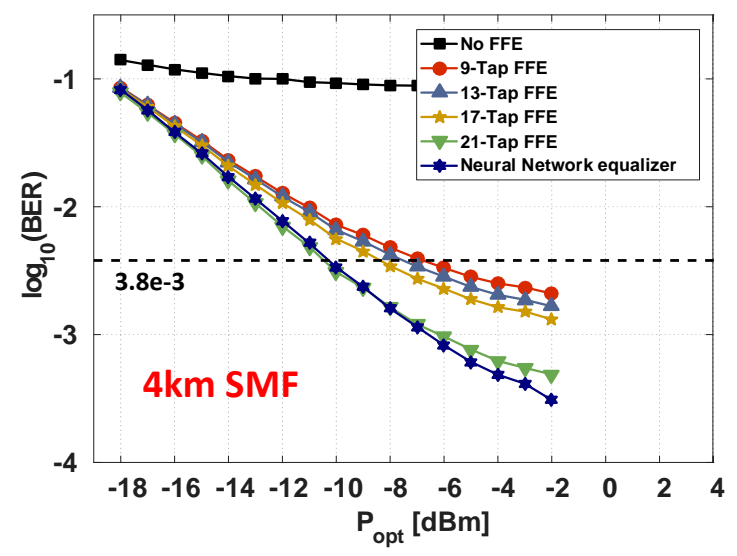

(b)

Fig. 3 Measured BER performances as a function of received optical power for 32GBd PAM-8 before and after 4km SMF transmission with different digital equalization implementations.

signal-to-noise ratio (SNR). Four implementations of linear equalizer are evaluated, namely, 9-, 13,-, 17- and 21-tap FFE, in addition to the neural network-based equalizer described above.

In the B2B case, both the FFE and the NNE successfully equalize the signal to BER levels well below the 7\%$\mathrm{OH}$ HD-FEC threshold. The performance improves with increasing the tap numbers for the FFE cases. The converged NNE clearly outperforms the FFE with lower than 21 taps and achieves slight better performance than the 21-tap FFE at high received optical power. At lower optical input power the noise starts to dominate so the BER performances converge between the 21-tap FFE and the NNE. The bending of the BER curves at $-6 \mathrm{dBm}$ is due to measurement error.

Equalization performances of different implementations of FFE and the proposed Neural Network equalizer are respectively presented. Similar performances are observed after $4 \mathrm{~km} \mathrm{SMF}$ transmission. Without any digital equalization the signal cannot be recovered due to strong ISI as shown in eyediagram in Fig.2. Around $2 \mathrm{~dB}$ sensitivity degradation is induced by fiber transmission for all equalization cases by comparing with the B2B. The NNE results in a better performance than the 21-tap FFE at high received power, same as the B2B case, while converge with each other at low Rx power when limited by noise.

\section{Conclusion}

We experimentally demonstrated a 4 km 32 GBd PAM-8 IM/DD link with digital equalization based on neural network. We show that the NN-based equalizer is able to accurately reconstruct the received signal suffering from strong ISI. Enhanced equalization performance of the NNE is observed comparing to the conventional linear FFE with as many as 21 taps. This study indicates that NNE has the potential to be applied for system implementation in optical interconnects and is worthy of further detailed explorations.

\section{Acknowledgement}

This work is supported by EU project GRIFFON, gr. \#324391, EU project ICONE, gr. \#608099 and Swedish ICTTNG program. The equipment was funded by Knut and Alice Wallenberg foundation.

\section{References}

[1]. S. Kanazawa et al., "Transmission of 214-Gbit/s 4-PAM signal using an ultra-broadband lumped-electrode EADFB laser module," Proc. OFC, PDP Th5B.3, Anaheim (2016).

[2]. X. Pang et al., "Evaluation of High-Speed EML-based IM/DD links with PAM Modulations and Low-Complexity Equalization," Proc. ECOC, Poster Session, Dusseldorf (2016).

[3]. G. Katz, D. Sadot, “Radial Basis Function Network Equalizer for Optical Communication OOK System,” J. Lightwave Technol. 25, 26312637 (2007).

[4]. M. Piels et al., "Laser Rate Equation-Based Filtering for Carrier Recovery in Characterization and Communication," J. Lightwave Technol. 33, 3271-3279 (2015).

[5]. D. Zibar, "Nonlinear impairment compensation using expectation maximization for dispersion managed and unmanaged PDM 16-QAM transmission," Opt. Express 20, B181-B196 (2012).

[6]. I. Lyubomirsky, "Machine Learning Equalization Techniques for High Speed PAM4 Fiber Optic Communication Systems," CS229 Final Project Report, Stanford University, 2015.

[7]. O. Ozolins et al., "100 GHz EML for High Speed Optical Interconnect Applications,” Proc. ECOC, M2.4, Dusseldorf (2016). 